
\title{
$\begin{array}{ll}\text { Research Square } & \begin{array}{l}\text { Preprints are preliminary reports that have not undergone peer review. } \\ \text { They should not be considered conclusive, used to inform clinical practice, } \\ \text { or referenced by the media as validated information. }\end{array}\end{array}$
}

\section{A Selected Population Study Reveals the Biochemical Mechanism of Intramuscular Fat Deposition in Chicken Meat}

\section{Huanxian Cui}

Chinese Academy of Agricultural Sciences Institute of Animal Science

Lu Liu

Chinese Academy of Agricultural Sciences Institute of Animal Science

Xiaojing Liu

Chinese Academy of Agricultural Sciences Institute of Animal Science

Yongli Wang

Chinese Academy of Agricultural Sciences Institute of Animal Science

Na Luo

Chinese Academy of Agricultural Sciences Institute of Animal Science

\section{Xiaodong Tan}

Chinese Academy of Agricultural Sciences Institute of Animal Science

\section{Yuting Zhu}

Chinese Academy of Agricultural Sciences Institute of Animal Science

\section{Ranran Liu}

Chinese Academy of Agricultural Sciences Institute of Animal Science

\section{Guiping Zhao}

Chinese Academy of Agricultural Sciences Institute of Animal Science

Jie Wen ( $\sim$ wenjie@caas.cn )

Institute of Animal Sciences, Chinese Academy of Agricultural Sciences https://orcid.org/0000-0001-7460-4219

\section{Research}

Keywords: Intramuscular fat, Fatty acid composition, Biochemical mechanism, Chicken

Posted Date: November 19th, 2021

DOI: https://doi.org/10.21203/rs.3.rs-1049686/v1

License: (ㄷ) This work is licensed under a Creative Commons Attribution 4.0 International License. Read Full License 


\section{Abstract \\ Background}

Increasing intramuscular fat (IMF) is an important strategy to improve meat quality, but the regulation mechanism of IMF deposition needs to be systematically clarified.

\section{Results}

A total of 520 chickens from a selected line with improved IMF content and a control line were used to investigate the biochemical mechanism of IMF deposition in chickens. The results showed that the increased IMF would improve the flavor and tenderness quality of chicken meat. IMF content was mainly determined both by measuring triglyceride (TG) and phospholipid (PLIP) in muscle tissue, but only TG content was found to be decisive for IMF deposition. Furthermore, the increase in major fatty acid (FA) components in IMF is mainly derived from TGs (including C16:0, C16:1, C18:1n9c, and C18:2n6c, etc.), and the inhibition of certain very-long-chain FAs would help to IMF/TG deposition.

\section{Conclusions}

Our study elucidated the underlying biochemical mechanism of IMF deposition in chicken: Prevalent accumulation of long-chain FAs and inhibitions of medium-chain FAs and very long chain-FA would synergistically result in the increase of TGs with the FA biosynthesis and cellular uptake ways. Our findings will guide the production of high-quality chicken meat.

\section{Background}

As a result of its deliciousness, tenderness, and juicy quality, chicken is the second most-consumed meat worldwide by humans. With the use of continuous growth breeding and high-density feeding to produce more meat, the meat quality deteriorated due to the relatively higher water and lower fat content in the meat. Currently, the challenges of improving meat quality are the focus of intensive scientific research [1, 2]. Intramuscular fat (IMF), a mixture of various lipids (including triglyceride (TG), phospholipid (PLIP), total cholesterols (TCHO), etc.) in muscle tissue, which has an important role in determining the flavor, tenderness, and water holding capacity of meat [3-6]. For example, Kobe beef is known for its high meat quality grade which is determined by its high beef marbling (IMF) score (BMS).

Fatty acids (FAs), as a basic component of cells by forming TG and PLIP, besides being important structural components for cells, play an important regulatory role in animal biological processes [7-9], including the energy storage, information transfer and regulation of metabolism. The sources of FAs in animal tissues include cellular uptake and de novo lipogenesis $(D N L)$. FAs for cellular uptake are derived from blood lipids through lipoprotein lipase (LPL)-mediated hydrolysis $[10,11]$. However, carbon-16 FA (palmitic acid) is synthesized through the intracellular DNL pathway in a reaction catalyzed by FA synthase (FASN) using acetyl coenzyme $A$ and malonyl coenzyme $A$ as substrates, and then the carbon chain elongation is regulated by multiple proteins [12].

Due to the absence of the marbling typically found in chicken meat, the deposition of IMF in chicken cannot be directly identified anatomically and is thus difficult to accurately determine. These factors have greatly restricted the characterization of IMF deposition in chicken. As it is well known, DNL mainly occurs in the liver in poultry [13], and the necessary FAs are mainly provided by cellular uptake in other tissues. Moreover, in general, the muscle tissue mainly includes myocyte, adipocyte and connective tissue cells. Unlike abdominal fat, the formation of IMF is more complicated 
due to the interference of myocytes, thus more research is required for the elucidation of the underlying biochemical mechanism of IMF deposition in chickens.

A useful experimental model for elucidating the biochemical mechanism of IMF formation in chickens is advantageous $[14,15]$. Our research group had artificially bred a selected population of Jingxing yellow-feather chickens (JXY, which is a synthetic line derived from a local Chinese chicken) with the IMF content in breast tissue as the main selection trait [15]. In this study, we used this selected line at generation 16 and the corresponding control line of JXY chickens to systematically investigate the biochemical mechanism of IMF deposition. It is anticipated that our research will not only enrich the theory on IMF formation, but will also provide guidance for future production of high-quality broilers.

\section{Materials And Methods}

\section{Ethics statement}

This study was conducted in accordance with the Guidelines for Use of Experimental Animals established by the Ministry of Science and Technology (Beijing, China). All experimental protocols were approved by the Science Research Department (in charge of animal welfare issues) of the Institute of Animal Sciences, Chinese Academy of Agricultural Sciences (CAAS; Beijing, China) (No. IAS2019-21).

\section{Animals}

A total of 520 female JXY chickens (Selected line: $n=256$; Control line: $n=264$ ) obtained from the Institute of Animal Science, CAAS (Beijing, China) were used in this study. The two lines of JXY chickens originated from the same base population of JXY100, the selected line was selected for increased IMF with IMF in breast muscle tissue as the main selection trait, and the control line was randomly bred as previously described[15].

\section{Sample collection}

All birds were raised in three-stair step cages (one bird per cage) under the same nutritional condition, and euthanized under carbon dioxide anesthesia by severing the carotid artery at 98 days of age. Then, the breast muscle tissue was dissected and stored at $-80^{\circ} \mathrm{C}$ for subsequent analysis.

\section{IMF, TG, PLIP and TCHO measurement}

IMF content (\%) of breast muscle tissue samples of the $520 \mathrm{JXY}$ chickens was determined by extraction with petroleum ether in a Soxhlet apparatus [16] and expressed as the percentage of the dry weight of breast muscle tissue. A 2.0-g sample of each breast muscle tissue was homogenized and extracted by the method of Folch[17], then TG, PLIP, and $\mathrm{TCHO}$ contents were measured using commercially available kits (Beijing Deliman Biochemical Technology Co. Ltd., Beijing, China).

\section{TG and PLIP separation}

Samples of $6.0 \mathrm{~g}$ of breast muscle tissue from each of the $8 \mathrm{JXY}$ chickens in the groups with high- or low-IMF content were used for the isolation of TG and PLIP. First, total lipids were extracted by a reported method [17]. Subsequently, TG and PLIP were obtained in turn as previously described [18]. Briefly: a $0.5 \mathrm{~mL}$ aliquot of the lipid dissolved in $1 \mathrm{~mL}$ of chloroform was placed into an activated aminopropyl silica column, and $2 \mathrm{~mL}$ of chloroform-isopropanol solution (volume ratio, 2:1) was added to the column to obtain neutral lipids (mainly TG). Then, $3 \mathrm{~mL}$ of $2 \%$ acetic acid-diethyl ether (mass to mass ratio) was added to the column to elute the free FA. Finally, $3 \mathrm{~mL}$ of methanol was added to elute PLIP. After blowing nitrogen, the obtained TG and PLIP were used for the determination of the FA composition. 


\section{FA composition analysis}

A 5.0-g breast muscle tissue sample of each of the 520 JXY chickens, or the separated TG and PLIP were freeze-dried and ground for extraction and methylation of FAs. Then, the FA composition was determined by gas chromatography (GC) according to a method previously reported [19] using an HP6890 gas chromatograph (Agilent Technologies, Santa Clara, CA, USA).

\section{Meat quality index determination}

The meat color, tenderness, and volatile substance of the breast muscle tissue samples of each of the 520 JXY chickens, were determined by a previously described method $[15,19,20]$. Additionally, the flavor quality of the breast muscle tissue samples from each of the 3 birds of the selected line and control line was determined using the electronic nose technology according to a previously described method [21].

\section{Cells separation and treatment}

The different-type primary cells in the pectoralis major muscle of 7-day-old chickens were isolated to obtain the muscle satellite cells and mature adipocyte, using a method based in part on a previous report [22]. Additionally, the mature adipocyte layer was seeded into a $25-\mathrm{cm}^{2}$ cell culture flask containing complete medium. The flask was incubated inverted for 6 days enabling the adipocyte to attach to the upper surface and dedifferentiate, and subsequently reinverted for another 6 days to obtain preadipocytes $[23,24]$. Two types of cells were incubated in a humidified atmosphere of $5 \% \mathrm{CO}_{2}$ at $37^{\circ} \mathrm{C}$, and the passage 2 cells were seeded into 6 -well plates. Two days after reaching $100 \%$ confluence, all cells were harvested and used for the analysis of TG, PLIP and TCHO contents.

\section{RNA sequencing and analysis}

Our previous RNA sequencing data (Accession number CRA001908; http://bigd.big.ac.cn/gsa.) of pectoralis from each 8 individuals in the groups with high- or low-IMF content from 520 JXY chickens as previously reported [25] was used in this study. Gene expression levels were determined using the RPKM method. Differentially expressed genes (DEGs) between the two lines were analyzed using the edgeR in the R package and screened by the following criteria: $\mid \log 2 \mathrm{FCl} \geq$ 0.58 , with Padj< 0.05 .

\section{Statistical Analyses}

The significance of the differences between groups was tested by the Student t-test using the SPSS Version 22.0 (IBM Corp., Armonk, NY, USA). Confidence limits were set at $95 \%$, and $P<0.05\left(^{*}\right)$ or $P<0.01$ (**) were considered statistically significant. Data are presented as the mean \pm standard error of mean (SEM). The principal component analysis (PCA) and Spearman correlation analysis were performed in R statistical software (version 3.6.1).

\section{Results}

\section{Increase of IMF content improves the quality of chicken meat}

As expected, the results of the analysis of the 520 female JXY chickens from the selected line $(n=256)$ at generation 16 and control line $(n=264)$ showed that the IMF content was more prominently increased in the selected line than that in the control line $(P<0.01)$ (Fig. 1a). In addition, evaluation of the impact of the increased IMF content on the quality of breast meat revealed that the shear force of the breast meat of the selected line was significantly lower than that of the control line (Fig. 1b). Similarly, the contents of two important volatile substances, namely hexanal and heptanal, in the 
breast meat of the selected line were significantly higher $(P<0.01, P<0.05$, respectively) than those of the control line (Fig. 1c). However, the meat color of the breast meat was not significantly different between the two lines (Fig. 1d).

\section{Tg Controls The Imf Content In Chicken Meat}

The results on the contents of the main IMF components (TG, PLIP and TCHO) showed that PLIP content was the highest in IMF, and the contents of PLIP and TG were dominant relative to cholesterol that of TCHO (Fig. 2a). Another perspective, the contents of the main IMF components (TG, PLIP and TCHO) between the selected line and the control line revealed, as shown in Fig. 2b, that the contents of PLIP and TG were significantly higher in the breast muscle tissue of the selected line those in the control line $(P<0.01, P<0.05$, respectively), but the increase of the TG content was higher than that of PLIP. However, the TCHO content was not significantly different between the two lines $(P>0.05)$. Furthermore, Spearman's correlation analysis showed that the IMF content had a higher positive correlation with the TG content $(r=$ $0.45, P<0.01)$, and a relatively weak positive correlation with the PLIP content $(r=0.11, P<0.05)$, but not with the TCHO content (Fig. 2c and Supplemental Table 1). In addition, the determination of the contents of TG, PLIP and TCHO in myocytes and adipocytes from the pectoral muscle tissue revealed that in adipocytes the TG content was higher $(P<$ $0.01, P<0.01)$ than those of PLIP and TCHO, while in myocytes the PLIP content was higher $(P<0.01, P<0.01)$ than the TG and TCHO contents (Fig. 2d).

\section{Change Of Fa Composition Determines Imf Deposition}

A total of 23 common FAs in breast muscle tissue were commonly shared by both the selected line and control line, and the $\mathrm{C} 16: 0, \mathrm{C} 18: 0, \mathrm{C} 18: 1 \mathrm{n} 9 \mathrm{c}, \mathrm{C} 18: 2 \mathrm{n} 6 \mathrm{c}$ and $\mathrm{C} 20: 4 \mathrm{n} 6$ are considered as the main structural components with a proportion exceeding 10\% (Table 1). Additionally, the results of the PCA showed that C16:0, C16:1, C18:0, C18:1n9c, C18:2n6c and $\mathrm{C} 20: 4 \mathrm{n} 6$ were represented in the first principal component, as shown in Fig. 3, suggesting that these long-chain FAs are dominant in the process of IMF deposition. Among 23 common FAs, the proportions of C14:0, C16:1, C18:1n9c and C18:3n3 were significantly increased $(P<0.05$ or $P<0.01)$, but those of C10:0, C18:0, C20:0, C20:3n6, C20:4n6 and C22:6n3 were significantly decreased $(P<0.05$ or $P<0.01)$ in the selected line compared to those in the control line (Table 1). In addition, the correlation analysis found positive correlations between TG/IMF and important long-chain FAs (mainly including C14:0, C14:1, C16:0, C16:1, C18:1n9c and C18:3n3), and negative correlations between TG/IMF and other FAs (mainly including C18:0, C20:0, C21:0, C22:0, C20:3n6, C20:4n6, C20:5n3, C22:6n3, C24:0 and C24:1) (Fig. 4 and Supplemental Table 2). 
Table 1

Comparison of fatty acid composition in muscle tissue of the selected line and control line of JXY chicken.

\begin{tabular}{|c|c|c|}
\hline Fatty acid (\%) & Selected line & Control line \\
\hline C10:0 & $0.99 \pm 0.24^{b}$ & $1.05 \pm 0.28^{a}$ \\
\hline C12:0 & $0.31 \pm 0.11$ & $0.32 \pm 0.10$ \\
\hline C14:0 & $0.34 \pm 0.05^{a}$ & $0.31 \pm 0.05^{b}$ \\
\hline C14:1 & $0.04 \pm 0.01$ & $0.04 \pm 0.01$ \\
\hline C15:0 & $0.06 \pm 0.01$ & $0.06 \pm 0.01$ \\
\hline C16:0 & $23.84 \pm 0.61$ & $23.75 \pm 0.62$ \\
\hline C16:1 & $1.30 \pm 0.25^{a}$ & $1.21 \pm 0.24^{b}$ \\
\hline C17:0 & $0.13 \pm 0.02$ & $0.14 \pm 0.01$ \\
\hline C18:0 & $12.74 \pm 0.61^{b}$ & $12.97 \pm 0.67^{a}$ \\
\hline C18:1n9c & $26.25 \pm 1.99^{a}$ & $25.39 \pm 2.13^{b}$ \\
\hline C18:2n6c & $17.73 \pm 1.11$ & $17.56 \pm 1.01$ \\
\hline C18:3n3 & $0.60 \pm 0.09^{a}$ & $0.58 \pm 0.09^{b}$ \\
\hline C20:0 & $0.17 \pm 0.02^{b}$ & $0.17 \pm 0.03^{a}$ \\
\hline C20:1 & $0.23 \pm 0.04$ & $0.23 \pm 0.06$ \\
\hline C21:0 & $0.67 \pm 0.14$ & $0.69 \pm 0.15$ \\
\hline C20:3n6 & $0.98 \pm 0.13^{b}$ & $1.01 \pm 0.14^{\mathrm{a}}$ \\
\hline C20:4n6 & $10.26 \pm 1.36^{b}$ & $10.96 \pm 1.42^{a}$ \\
\hline C20:5n3 & $0.15 \pm 0.03$ & $0.16 \pm 0.03$ \\
\hline C22:0 & $0.19 \pm 0.06$ & $0.19 \pm 0.05$ \\
\hline C22:1n9 & $0.05 \pm 0.02$ & $0.05 \pm 0.03$ \\
\hline $\mathrm{C} 24: 0$ & $1.40 \pm 0.27$ & $1.54 \pm 0.27$ \\
\hline C22:6n3 & $1.22 \pm 0.24^{b}$ & $1.30 \pm 0.24^{\mathrm{a}}$ \\
\hline C24:1 & $0.30 \pm 0.10$ & $0.30 \pm 0.10$ \\
\hline
\end{tabular}

\section{FAs in TG determine the total FAs composition of IMF}

The breast muscle tissue of each 8 individuals in the groups with high- or low-IMF content were also used to separately extract TG and PLIP to determine the FA composition of IMF. As shown in Table 2, the proportions of C14:0, C16:0, C16:1, C18:1n9c, C18:3n3, and C20:1 were significantly higher, while the proportions of C18:0, C20:0, C20:4n6, C20:4n6, C20:5n3, C22:0, C21:1n9, and C24:0 were significantly $(P<0.05$ or $P<0.01)$ lower in TGs of the high-IMF group than those of the low-IMF group, which was consistent with the changes of C14:0, C16:0, C16:1, C18:1n9c, C18:3n3, and C20:1 
proportions in breast muscle tissue between the high-IMF and low-IMF group. However, only the proportions of C12:0 and C15:0 were significantly different $(P<0.05)$, and the proportions of the remaining FAs did not show significant changes of PLIP in the high-IMF group compared to those in the low-IMF group $(P>0.05)$.

Table 2

Fatty acid composition in separately extract IMF, TG and PLIP from muscle tissue of JXY chicken with high- or low-IMF content.

\begin{tabular}{|c|c|c|c|c|c|c|}
\hline \multirow{2}{*}{$\begin{array}{l}\text { Fatty } \\
\text { acid (\%) }\end{array}$} & \multicolumn{2}{|l|}{ IMF } & \multicolumn{2}{|l|}{ TG } & \multicolumn{2}{|l|}{ PL } \\
\hline & High IMF & Low IMF & High IMF & Low IMF & High IMF & Low IMF \\
\hline C12:0 & $0.278 \pm 0.097^{b}$ & $0.394 \pm 0.145^{a}$ & $0.059 \pm 0.029$ & $0.060 \pm 0.022$ & $0.046 \pm 0.007^{b}$ & $0.055 \pm 0.009^{a}$ \\
\hline C14:0 & $0.374 \pm 0.066^{a}$ & $0.286 \pm 0.052^{b}$ & $0.314 \pm 0.056^{a}$ & $0.232 \pm 0.028^{b}$ & $0.133 \pm 0.023$ & $0.148 \pm 0.038$ \\
\hline C15:0 & $0.060 \pm 0.008$ & $0.067 \pm 0.015$ & $0.057 \pm 0.009$ & $0.058 \pm 0.012$ & $0.086 \pm 0.024^{b}$ & $0.116 \pm 0.025^{a}$ \\
\hline C16:0 & $24.128 \pm 0.720^{a}$ & $23.418 \pm 0.591^{b}$ & $28.064 \pm 1.375$ & $28.262 \pm 1.625$ & $25.934 \pm 1.300$ & $26.981 \pm 1.532$ \\
\hline C16:1 & $1.583 \pm 0.189^{a}$ & $1.029 \pm 0.210^{b}$ & $1.192 \pm 0.229^{a}$ & $0.741 \pm 0.179^{b}$ & $0.249 \pm 0.051$ & $0.257 \pm 0.054$ \\
\hline C17:0 & $0.135 \pm 0.016$ & $0.129 \pm 0.015$ & $0.104 \pm 0.039$ & $0.106 \pm 0.018$ & $0.116 \pm 0.02$ & $0.132 \pm 0.020$ \\
\hline C18:0 & $11.633 \pm 0.438^{b}$ & $13.901 \pm 0.719^{a}$ & $9.546 \pm 0.513^{b}$ & $11.163 \pm 0.713^{a}$ & $13.612 \pm 0.898$ & $13.866 \pm 0.670$ \\
\hline C18:1n9c & $29.443 \pm 1.373^{\mathrm{a}}$ & $22.831 \pm 1.682^{\mathrm{b}}$ & $26.227 \pm 1.979^{a}$ & $21.262 \pm 1.706^{b}$ & $19.290 \pm 1.523$ & $18.780 \pm 1.345$ \\
\hline C18:2n6c & $18.009 \pm 1.165$ & $17.341 \pm 1.309$ & $17.616 \pm 1.369$ & $17.316 \pm 1.267$ & $13.131 \pm 1.319$ & $14.135 \pm 1.116$ \\
\hline C18:3n3 & $0.750 \pm 0.084^{a}$ & $0.471 \pm 0.090^{b}$ & $0.518 \pm 0.132^{\mathrm{a}}$ & $0.298 \pm 0.070^{b}$ & $0.049 \pm 0.010$ & $0.052 \pm 0.015$ \\
\hline C20:0 & $0.147 \pm 0.012^{b}$ & $0.204 \pm 0.028^{a}$ & $0.193 \pm 0.027^{b}$ & $0.233 \pm 0.049^{a}$ & $0.241 \pm 0.031$ & $0.230 \pm 0.026$ \\
\hline C20:1 & $0.246 \pm 0.018^{a}$ & $0.193 \pm 0.030^{b}$ & $0.182 \pm 0.035^{a}$ & $0.153 \pm 0.017^{b}$ & $0.108 \pm 0.011$ & $0.102 \pm 0.012$ \\
\hline C21:0 & $0.552 \pm 0.056^{b}$ & $0.752 \pm 0.041^{a}$ & $0.512 \pm 0.044^{b}$ & $0.636 \pm 0.052^{\mathrm{a}}$ & $0.586 \pm 0.054$ & $0.587 \pm 0.071$ \\
\hline C20:3n6 & $0.789 \pm 0.093^{b}$ & $1.138 \pm 0.105^{a}$ & $0.998 \pm 0.174^{b}$ & $1.226 \pm 0.113^{a}$ & $1.202 \pm 0.127$ & $1.185 \pm 0.127$ \\
\hline C20:4n6 & $8.206 \pm 0.841^{b}$ & $12.329 \pm 1.363^{a}$ & $9.402 \pm 1.694^{b}$ & $11.959 \pm 0.857^{a}$ & $18.775 \pm 1.519$ & $17.468 \pm 1.517$ \\
\hline C20:5n3 & $0.128 \pm 0.022^{b}$ & $0.177 \pm 0.023^{a}$ & $0.142 \pm 0.028^{b}$ & $0.170 \pm 0.029^{a}$ & $0.299 \pm 0.028$ & $0.274 \pm 0.046$ \\
\hline $\mathrm{C} 22: 0$ & $0.178 \pm 0.088^{b}$ & $0.219 \pm 0.041^{a}$ & $0.232 \pm 0.045$ & $0.273 \pm 0.044$ & $0.264 \pm 0.058$ & $0.239 \pm 0.023$ \\
\hline C21:1n9 & $0.039 \pm 0.018^{b}$ & $0.073 \pm 0.028^{a}$ & $1.994 \pm 0.319^{b}$ & $2.425 \pm 1.094^{a}$ & $0.078 \pm 0.015$ & $0.079 \pm 0.014$ \\
\hline $\mathrm{C} 24: 0$ & $1.098 \pm 0.149^{b}$ & $1.735 \pm 0.249^{a}$ & $1.146 \pm 0.201^{b}$ & $1.540 \pm 0.165^{a}$ & $2.531 \pm 0.357$ & $2.433 \pm 0.428$ \\
\hline C22:6n3 & $0.994 \pm 0.192^{b}$ & $1.510 \pm 0.168^{a}$ & $1.126 \pm 0.248$ & $1.465 \pm 0.138$ & $2.875 \pm 0.398$ & $2.524 \pm 0.394$ \\
\hline C24:1 & $0.288 \pm 0.169$ & $0.390 \pm 0.176$ & $0.376 \pm 0.065$ & $0.421 \pm 0.126$ & $0.394 \pm 0.066$ & $0.358 \pm 0.031$ \\
\hline
\end{tabular}

FA synthesis and extracellular intake are jointly involved in IMF deposition 
As shown in Fig. 4, the results of the correlation analysis performed to investigate the sources of FAs revealed a higher positive correlation between any two FAs of C14:0, C14:1, C16:0, C16:1, C18:1n9c and C18:3n3. Also, using our previous RNA-seq data of breast muscle tissue from each 8 individuals in the groups with high- or low-IMF content, some DEGs related to FA synthesis were screened by the following criteria: $\mid \log 2 \mathrm{FCl} \geq 0.58$, with $P a d j<0.05$. These DEGs are mainly involved in multiple processes of FA metabolism, including the processes of DNL (FASN, SRFBP1), release ( $L P L)$, desaturation (SCD5), elongation (ELOVL5, ELOVL7), transport (FABP4, FABP5, FABP9, CD36) and activation (ACSL5) of FAs. The expression levels of all these genes were significantly $(P<0.01)$ up-regulated in the high-IMF content group compared to those in the low-IMF content group (Fig. 5).

\section{Discussion}

Chicken is the second most consumed meat worldwide by humans, and the quality of meat is also the important demand of people. In the last few years, IMF has received increasing attention due to its major effect on meat quality [26-28], with Kobe beef as a popular example of high-quality grade meat with a high BMS. Our previous studies also demonstrated that the increase of IMF improves the meat quality in JXY chickens $[25,29]$. However, the metabolic pathway of chicken IMF deposition is still unclear due to the limitation of the physiological characteristics of chickens. A selected line of JXY chickens has been bred to contain a high IMF content in breast muscle tissue [15], which is critical to the success of scientific research as the suitable experimental model [30]. As expected, we had explained the biochemical mechanism of IMF deposition in chicken in this study by using the populations of the selected line at generation 16 and control line.

A significant increase of the IMF content was confirmed in breast muscle tissue of the selected line at generation 16 compared with that in the control line. Also, the increased IMF was found to promote tenderness and flavor quality in chicken meat as previously reported [15], which confirmed the validity of this selected line model. IMF is a lipid mixture that accumulates in chicken muscle tissue, consisting mainly of TG, PLIP and TCHO [31]. First, the result revealed that the changes of TG content was consistent with the IMF content difference between the two lines, suggesting that the TG content has a more decisive effect on IMF deposition in chicken meat, which is supported by the results of the correlation analysis.

Different from adipocytes as the dominant cell type in abdominal fat tissue, myocytes had an absolute advantage in muscle tissue. Due to the limitations of the method for IMF separation, all lipids in the cells are extracted. PLIP is an essential component of cell membrane [32], so the obtained IMF actually contains the deposited TG in cytoplasm and the structural PLIP from the cytomembrane $[17,18]$. The results of in vitro experiments in this study showed that TGs were mainly derived from adipocytes, and a certain amount of PLIP were derived from myocytes in muscle tissue. Thus, we inferred that the TG content was decisive to IMF deposition, although TG and PLIP might together affect the IMF content.

FA is a structural component of TG and PLIP, and also an important precursor for the flavor volatile compounds [33]. Therefore, we conducted an in-depth investigation of the effect of FAs on the deposition of TG, PLIP, and IMF in the selected line and control line. The results on the FA composition revealed that the long chain FAs (LCFAs), including C16:0, C16:1, C18:0, C18:1n9c, C18:2n6c and C20:4n6, were more prevalent prevailing with a proportion exceeding 10\% in chicken meat as previously reported (Hoa et al., 2019; Luo et al., 2019), which can explain the $95.34 \%$ phenotypic variation determined by PCA analysis in this study.

Furthermore, the comparison of the selected line with the control line showed an increase of the C14:0, C16:1, C18:1n9c, C18:3n3 proportions with high positive correlations of TG/IMF, and a decrease of the C10:0, C12:0, C18:0, C20:0, C21:0, C22:0, C20:3n6, C20:4n6, C20:5n3, C22:6n3, C24:0, C24:1 with high negative correlations of TG/IMF. Since the increase of very long chain-FA (VLCFA) would reduce the deposition of TG [34,35], these results suggested that prevalent accumulation of LCFAs and inhibition of medium chain FAs (MCFAs) and VLCFAs synergistically resulted in the increase 
of TG/IMF in the selected line. Also, by separately isolating TG and PLIP using each eight individuals in the groups with the high- or low-IMF contents, we found that the FA composition of IMF is mainly determined by TG, not by PLIP.

The sources of FAs in animal tissues include cellular uptake and DNL. DNL mainly occurs in the liver in poultry [13], and the necessary FAs are mainly provided by cellular uptake in other tissues. In this study, we investigated anew the source of FAs in chicken muscle tissues. Using the above phenotypic correlation analysis based on the FA data of all 520 chickens, the high positive correlation between any two FAs of C14:0, C14:1, C16:0, C16:1, C18:1n9c and C18:3n3 suggested that DNL should occurs in chicken meat.

As an auxiliary means, the RNA-seq data of breast muscle tissue from each 8 individuals in the groups with high- or lowIMF content were used, and some DEGs related to DNL (FASN, SRFBP1), release ( $L P L)$, desaturation (SCD5), elongation (ELOVL5, ELOVL7), and activation (ACSL5) of FAs were screened. Among them, LPL has an important role on releasing FAs from blood lipid [10, 11], and ACSL5 specifically activates FAs from food sources [36], thus insuring the availability of FAs for the cellular uptake option in chicken muscle tissue. In addition, it is well established that FASN and SRFBP1 are involved in the regulation of the de novo synthesis of FAs [37, 38]. Similarly, SCD and ELOVLs have an important role in the desaturation or elongation of FAs [39].

Accordingly, it was inferred that FAs synthesis also occurs in chicken muscle. In combination with the above results, we mapped out the biochemical metabolic pathway of IMF deposition in chicken, as shown in Fig. 6.

\section{Conclusions}

In summary, our study elucidated the underlying biochemical mechanism of IMF deposition in chicken. Prevalent accumulation of LCFAs (C16:0, C16:1, C18:1n9c, and C18:2n6c), and inhibitions of MCFAs and VLCFAs would synergistically result in the increase of TGs with the FA biosynthesis and cellular uptake ways, and then accelerate the deposition of IMF in chicken meat. Our findings will contribute to improve our understanding of IMF occurrence in chicken, and will provide guidance for the production of high-quality chicken meat.

\section{Declarations}

\section{Acknowledgments}

The authors highly appreciate the excellent technical support by professor Daoying Wang and Dr. Xinxiao Zhang and their members in the Livestock and Poultry Products Processing Laboratory in Jiangsu Academy of Agricultural Science (Jiangsu, China).

\section{Authors' contributions}

Huanxian Cui: Writing-orignal draft \& review \& editing, Formal analysis, Project administration, Conceptualization. Lu Liu: Writing-orignal draft, Formal analysis, Methodology, Validation. Xiaojing Liu: Writing-orignal draft, Investigation, Validation. Yongli Wang: Writing-orignal draft, Resources. Na Luo: Writing-orignal draft, Validation. Xiaodong Tan: Writing-orignal draft. Jie Wang: Writing-orignal draft. Yuting Zhu: Writing-orignal draft. Ranran Liu: Writing-review \& editing. Guiping Zhao: Writing-review \& editing, Supervision, Conceptualization. Jie Wen: Writing-review \& editing, Supervision, Conceptualization.

\section{Funding}

This research was funded by grants from the National Natural Science Foundation of China (31872340), the Basic Research from Institute of Animal Sciences of Chinese Academy of Agricultural Sciences (2019-YWF-YB-07), the 
Agricultural Science and Technology Innovation Program (CAAS-ZDRW202005), the Basic Research from Chinese Academy of Agricultural Sciences (Y2019XK06), the Agricultural Science and Technology Innovation Program (ASTIPIAS04), and the China Agriculture Research System of MOF and MARA (CARS-41).

\section{Availability of data and materials}

All data generated or analyzed during this study are included in this published article.

\section{Declaration of competing interests}

The authors declare no competing interests.

\section{References}

1. Sun G, Li F, Ma X, Sun J, Jiang R, Tian Y, Han R, Li G, Wang Y, Li Z et al: gga-miRNA-18b-3p Inhibits Intramuscular Adipocytes Differentiation in Chicken by Targeting the ACOT13 Gene. Cells 2019, 8(6).https://doi.org/10.3390/cells8060556.

2. Li G, Zhao Y, Li Y, Chen Y, Jin W, Sun G, Han R, Tian Y, Li H, Kang X: Weighted gene coexpression network analysis identifies specific transcriptional modules and hub genes related to intramuscular fat traits in chicken breast muscle. J Cell Biochem 2019, 120(8):13625-13639.https://doi.org/10.1002/jcb.28636.

3. Wood JD, Enser M, Fisher AV, Nute GR, Sheard PR, Richardson RI, Hughes SI, Whittington FM: Fat deposition, fatty acid composition and meat quality: A review. Meat Sci 2008, 78(4):343358.https://doi.org/10.1016/j.meatsci.2007.07.019.

4. Inoue K, Shoji N, Honda T, Oyama K: Genetic relationships between meat quality traits and fatty acid composition in Japanese black cattle. Anim Sci J 2017, 88(1):11-18.https://doi.org/10.1111/asj.12613.

5. Huang Y, Zhou L, Zhang J, Liu X, Zhang Y, Cai L, Zhang W, Cui L, Yang J, Ji J et al: A large-scale comparison of meat quality and intramuscular fatty acid composition among three Chinese indigenous pig breeds. Meat Sci 2020, 168:108182.https://doi.org/10.1016/j.meatsci.2020.108182.

6. Sarsenbek A, Wang T, Zhao JK, Jiang W: Comparison of carcass yields and meat quality between Baicheng-You chickens and Arbor Acres broilers. Poult Sci 2013, 92(10):2776-2782.https://doi.org/10.3382/ps.2012-02841.

7. Levental KR, Malmberg E, Symons JL, Fan YY, Chapkin RS, Ernst R, Levental I: Lipidomic and biophysical homeostasis of mammalian membranes counteracts dietary lipid perturbations to maintain cellular fitness. Nat Commun 2020, 11(1):1339.https://doi.org/10.1038/s41467-020-15203-1.

8. Nakamura MT, Yudell BE, Loor JJ: Regulation of energy metabolism by long-chain fatty acids. Prog Lipid Res 2014, 53:124-144.https://doi.org/10.1016/j.plipres.2013.12.001.

9. Bazinet RP, Layé S: Polyunsaturated fatty acids and their metabolites in brain function and disease. Nat Rev Neurosci 2014, 15(12):771-785.https://doi.org/10.1038/nrn3820.

10. Aryal B, Singh AK, Zhang X, Varela L, Rotllan N, Goedeke L, Chaube B, Camporez JP, Vatner DF, Horvath TL et al: Absence of ANGPTL4 in adipose tissue improves glucose tolerance and attenuates atherogenesis. JCl Insight 2018, 3(6).https://doi.org/10.1172/jci.insight.97918.

11. Kersten S: Physiological regulation of lipoprotein lipase. Biochim Biophys Acta 2014, 1841(7):919933.https://doi.org/10.1016/j.bbalip.2014.03.013.

12. Roy R, Taourit S, Zaragoza P, Eggen A, Rodellar C: Genomic structure and alternative transcript of bovine fatty acid synthase gene (FASN): comparative analysis of the FASN gene between monogastric and ruminant species.

Cytogenet Genome Res 2005, 111(1):65-73.https://doi.org/10.1159/000085672. 
13. Hermier D: Lipoprotein metabolism and fattening in poultry. J Nutr 1997, 127(5 Suppl):805S808S.https://doi.org/10.1093/jn/127.5.805S.

14. Cui XY, Li Y, Liu RR, Zhao GP, Zheng MQ, Li QH, Wen J: Follicle-stimulating hormone increases the intramuscular fat content and expression of lipid biosynthesis genes in chicken breast muscle. Journal of Zhejiang University Science B 2016, 17(4)

15. Zhao GP, Chen JL, Zheng MQ, Wen J, Zhang Y: Correlated responses to selection for increased intramuscular fat in a Chinese quality chicken line. Poult Sci 2007, 86(11):2309-2314.https://doi.org/10.1093/ps/86.11.2309.

16. Hopkins DL, Clayton EH, Lamb TA, van de Ven RJ, Refshauge G, Kerr MJ, Bailes K, Lewandowski P, Ponnampalam EN: The impact of supplementing lambs with algae on growth, meat traits and oxidative status. Meat Sci 2014, 98(2):135-141.https://doi.org/10.1016/j.meatsci.2014.05.016.

17. Folch J, Lees M, Sloane Stanley GH: A simple method for the isolation and purification of total lipides from animal tissues. J Biol Chem 1957, 226(1):497-509

18. García Regueiro JA, Gibert J, Díaz I: Determination of neutral lipids from subcutaneous fat of cured ham by capillary gas chromatography and liquid chromatography. J Chromatogr A 1994, 667(1-2):225233.https://doi.org/10.1016/0021-9673(94)89071-4.

19. Tao F, Ngadi M: Recent advances in rapid and nondestructive determination of fat content and fatty acids composition of muscle foods. Crit Rev Food Sci Nutr 2018, 58(9):15651593.https://doi.org/10.1080/10408398.2016.1261332.

20. Shi Y, Li X, Huang A: A metabolomics-based approach investigates volatile flavor formation and characteristic compounds of the Dahe black pig dry-cured ham. Meat Sci 2019, 158:107904.https://doi.org/10.1016/j.meatsci.2019.107904.

21. Jin Y, Cui H, Yuan X, Liu L, Liu X, Wang Y, Ding J, Xiang H, Zhang X, Liu J et al: Identification of the main aroma compounds in Chinese local chicken high-quality meat. Food Chem 2021, 359:129930.https://doi.org/10.1016/j.foodchem.2021.129930.

22. Guo L, Cui H, Zhao G, Liu R, Li Q, Zheng M, Guo Y, Wen J: Intramuscular preadipocytes impede differentiation and promote lipid deposition of muscle satellite cells in chickens. BMC Genomics 2018, 19(1):838.https://doi.org/10.1186/s12864-018-5209-5.

23. Matsumoto T, Kano K, Kondo D, Fukuda N, Iribe Y, Tanaka N, Matsubara Y, Sakuma T, Satomi A, Otaki M et al: Mature adipocyte-derived dedifferentiated fat cells exhibit multilineage potential. J Cell Physiol 2008, 215(1):210222.https://doi.org/10.1002/jcp.21304.

24. Sun W, Wang H, Li Y, Zhou X, Teng Y, Chen J: Acquisition of pig intramuscular preadipocytes through dedifferentiation of mature adipocytes and establishment of optimal induction conditions. Genet Mol Res 2013, 12(4):5926-5936.https://doi.org/10.4238/2013.November.26.2.

25. Liu L, Liu X, Cui H, Liu R, Zhao G, Wen J: Transcriptional insights into key genes and pathways controlling muscle lipid metabolism in broiler chickens. BMC Genomics 2019, 20(1):863.https://doi.org/10.1186/s12864-019-6221-0.

26. Zerehdaran S, Vereijken AL, van Arendonk JA, van der Waaijt EH: Estimation of genetic parameters for fat deposition and carcass traits in broilers. Poult Sci 2004, 83(4):521-525.https://doi.org/10.1093/ps/83.4.521.

27. Nishimura T: The role of intramuscular connective tissue in meat texture. Anim Sci J 2010, 81(1):2127.https://doi.org/10.1111/j.1740-0929.2009.00696.x.

28. Starkey CP, Geesink GH, Collins D, Hutton Oddy V, Hopkins DL: Do sarcomere length, collagen content, pH, intramuscular fat and desmin degradation explain variation in the tenderness of three ovine muscles? Meat Sci 2016, 113:51-58.https://doi.org/10.1016/j.meatsci.2015.11.013. 
29. Liu L, Cui H, Xing S, Zhao G, Wen J: Effect of Divergent Selection for Intramuscular Fat Content on Muscle Lipid Metabolism in Chickens. Animals (Basel) 2019, 10(1).https://doi.org/10.3390/ani10010004.

30. Cui X, Liu R, Cui H, Zhao G, Zheng M, Li Q, Liu J, Liu Z, Wen J: Effects of caponization and ovariectomy on objective indices related to meat quality in chickens. Poult Sci 2017, 96(3):770-777.https://doi.org/10.3382/ps/pew346.

31. Scollan ND, Price EM, Morgan SA, Huws SA, Shingfield KJ: Can we improve the nutritional quality of meat? Proc Nutr Soc 2017, 76(4):603-618.https://doi.org/10.1017/s0029665117001112.

32. Murphy DJ: The biogenesis and functions of lipid bodies in animals, plants and microorganisms. Prog Lipid Res 2001, 40(5):325-438.https://doi.org/10.1016/s0163-7827(01)00013-3.

33. Arshad MS, Sohaib M, Ahmad RS, Nadeem MT, Imran A, Arshad MU, Kwon JH, Amjad Z: Ruminant meat flavor influenced by different factors with special reference to fatty acids. Lipids Health Dis 2018, 17(1):223.https://doi.org/10.1186/s12944-018-0860-z.

34. Luo Y, Wang B, Liu C, Su R, Hou Y, Yao D, Zhao L, Su L, Jin Y: Meat quality, fatty acids, volatile compounds, and antioxidant properties of lambs fed pasture versus mixed diet. Food Sci Nutr 2019, 7(9):27962805.https://doi.org/10.1002/fsn3.1039.

35. Hoa VB, Seong PN, Cho SH, Kang SM, Kim YS, Moon SS, Choi YM, Kim JH, Seol KH: Quality characteristics and flavor compounds of pork meat as a function of carcass quality grade. Asian-Australas J Anim Sci 2019, 32(9):1448-1457.https://doi.org/10.5713/ajas.18.0965.

36. Perez-Nunez I, Karaky M, Fedetz M, Barrionuevo C, Izquierdo G, Matesanz F, Alcina A: Splice-site variant in ACSL5: a marker promoting opposing effect on cell viability and protein expression. Eur J Hum Genet 2019, 27(12):18361844.https://doi.org/10.1038/s41431-019-0414-5.

37. Wallace M, Green CR, Roberts LS, Lee YM, McCarville JL, Sanchez-Gurmaches J, Meurs N, Gengatharan JM, Hover JD, Phillips SA et al: Enzyme promiscuity drives branched-chain fatty acid synthesis in adipose tissues. Nat Chem Biol 2018, 14(11):1021-1031.https://doi.org/10.1038/s41589-018-0132-2.

38. Han Y, Hu Z, Cui A, Liu Z, Ma F, Xue Y, Liu Y, Zhang F, Zhao Z, Yu Y et al: Post-translational regulation of lipogenesis via AMPK-dependent phosphorylation of insulin-induced gene. Nat Commun 2019, 10(1):623.https://doi.org/10.1038/s41467-019-08585-4.

39. Mihelic R, Winter H, Powers JB, Das S, Lamour K, Campagna SR, Voy BH: Genes controlling polyunsaturated fatty acid synthesis are developmentally regulated in broiler chicks. Br Poult Sci 2020, 61(5):508517.https://doi.org/10.1080/00071668.2020.1759788.

\section{Figures}



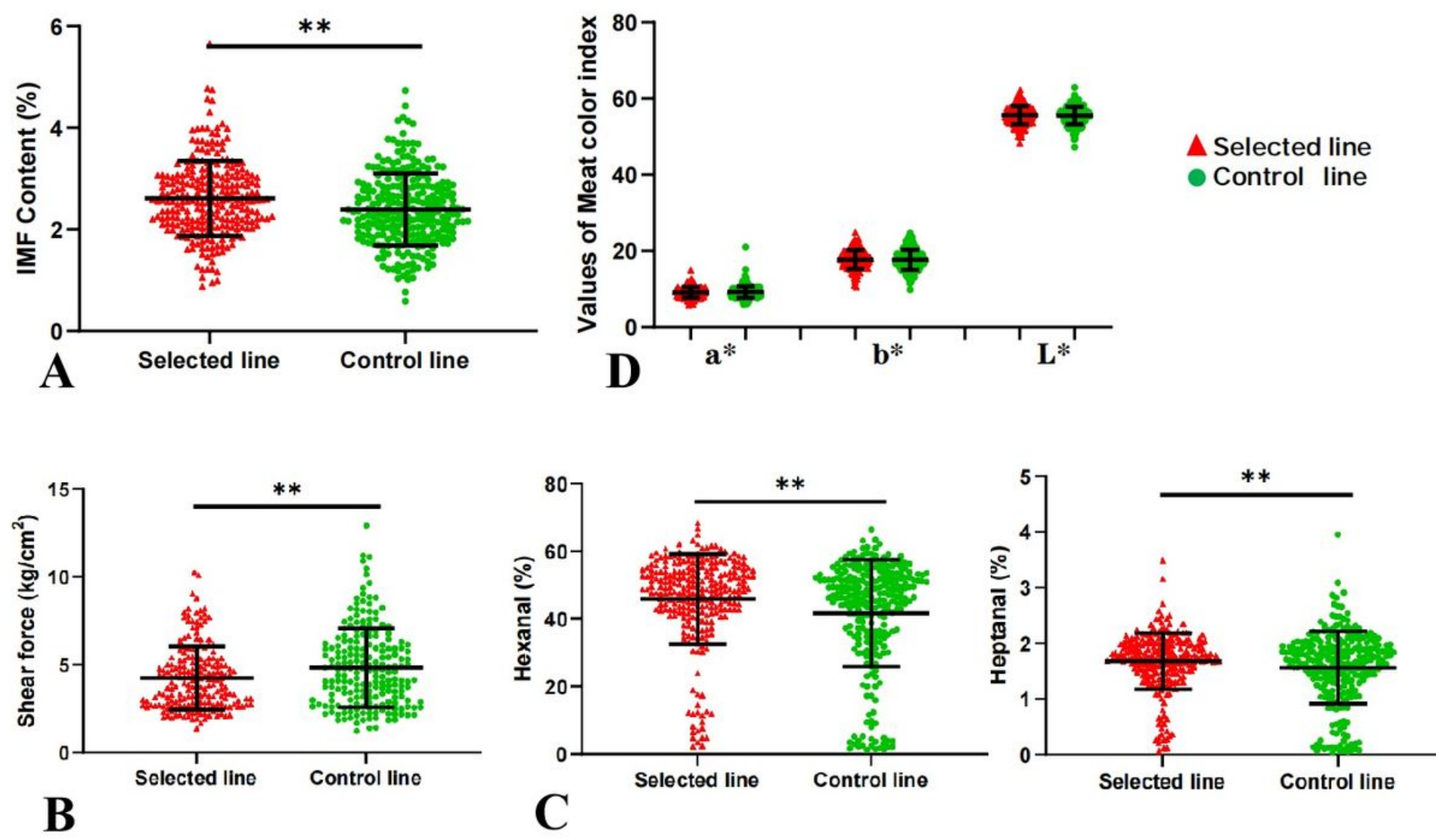

Figure 1

Comparison of IMF content and meat quality between the selected line and the control line. a. IMF content; b. Shear force; c. Contents of hexanal and heptanal; $d$. Meat color. Data are expressed as the mean \pm standard error of mean (SEM); Selected line: $n=256$; Control line: $n=264$. 

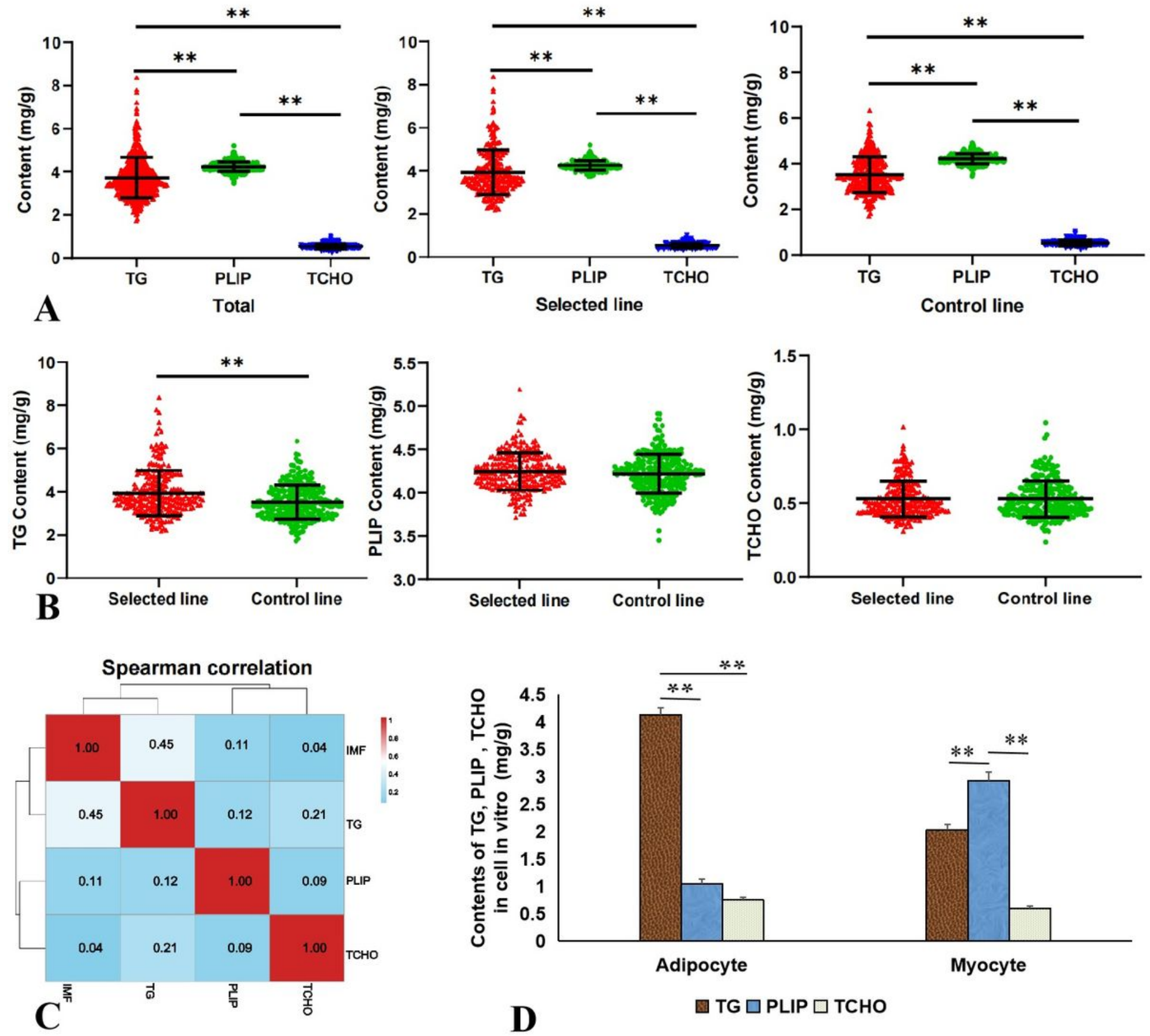

Figure 2

Identification of the decisive ingredients of IMF in chicken meat. a. Contents of TG, PLIP and CH in the selected line and the control line, respectively. b. Comparison of TG, PLIP and TCH contents between the selected line and the control line. c. Correlation analysis between IMF, TG, PLIP and TCH. d. Contents of TG, PLIP and TCH in myocytes or adipocytes derived from the pectoral muscle tissue. Data are expressed as the mean \pm standard error of mean (SEM); $n=520$ individual (in vivo) or 3 (in vitro). 


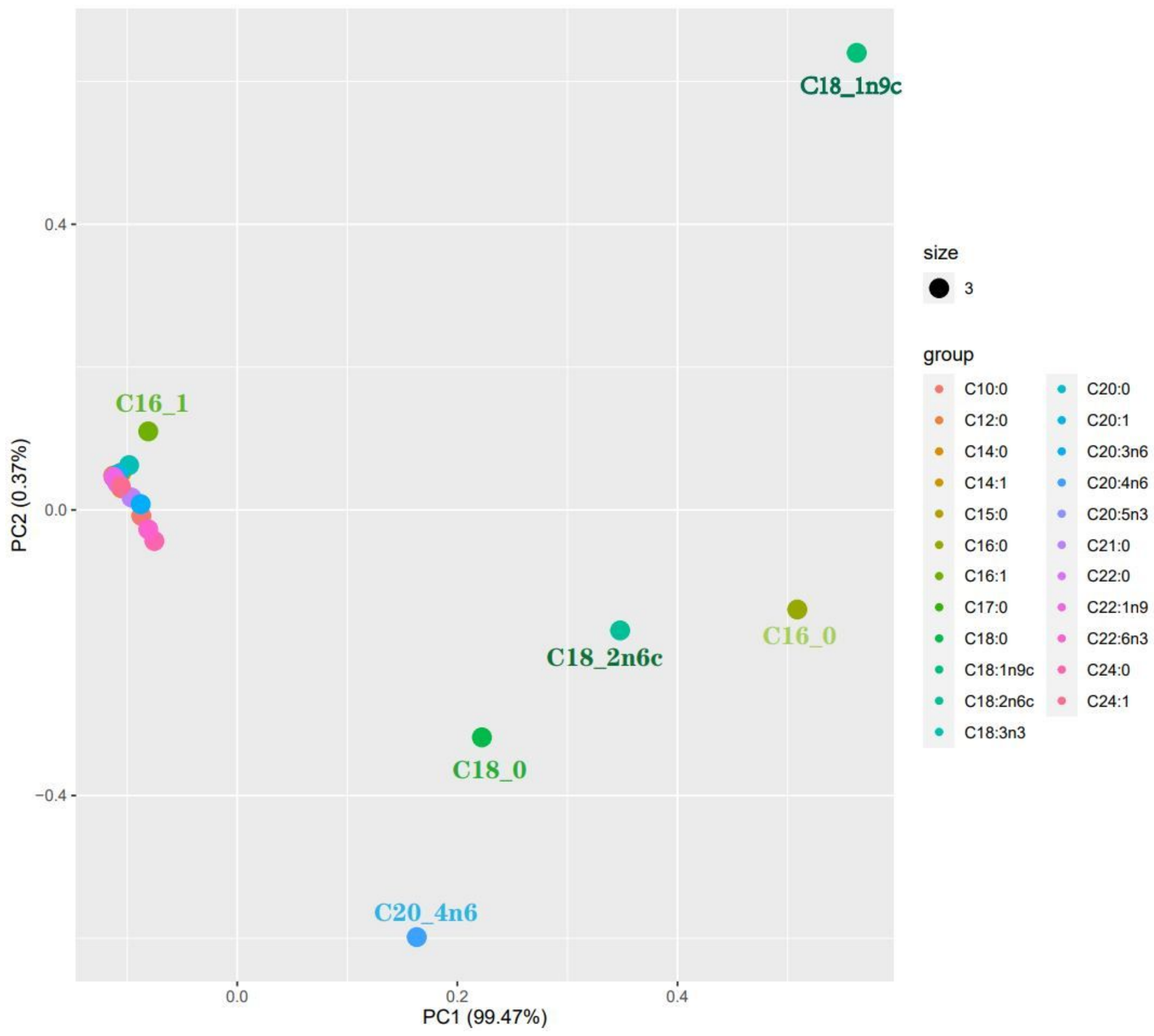

\section{Figure 3}

Principal components analysis (PCA) of fatty acid (FA) in chicken meat between the selected line and the control line. PCA based on data of FA composition of meat from all 520 chickens. Data are expressed as the mean \pm standard error of mean (SEM); Selected line: $n=256$; Control line: $n=264$. 


\section{Spearman correlation}

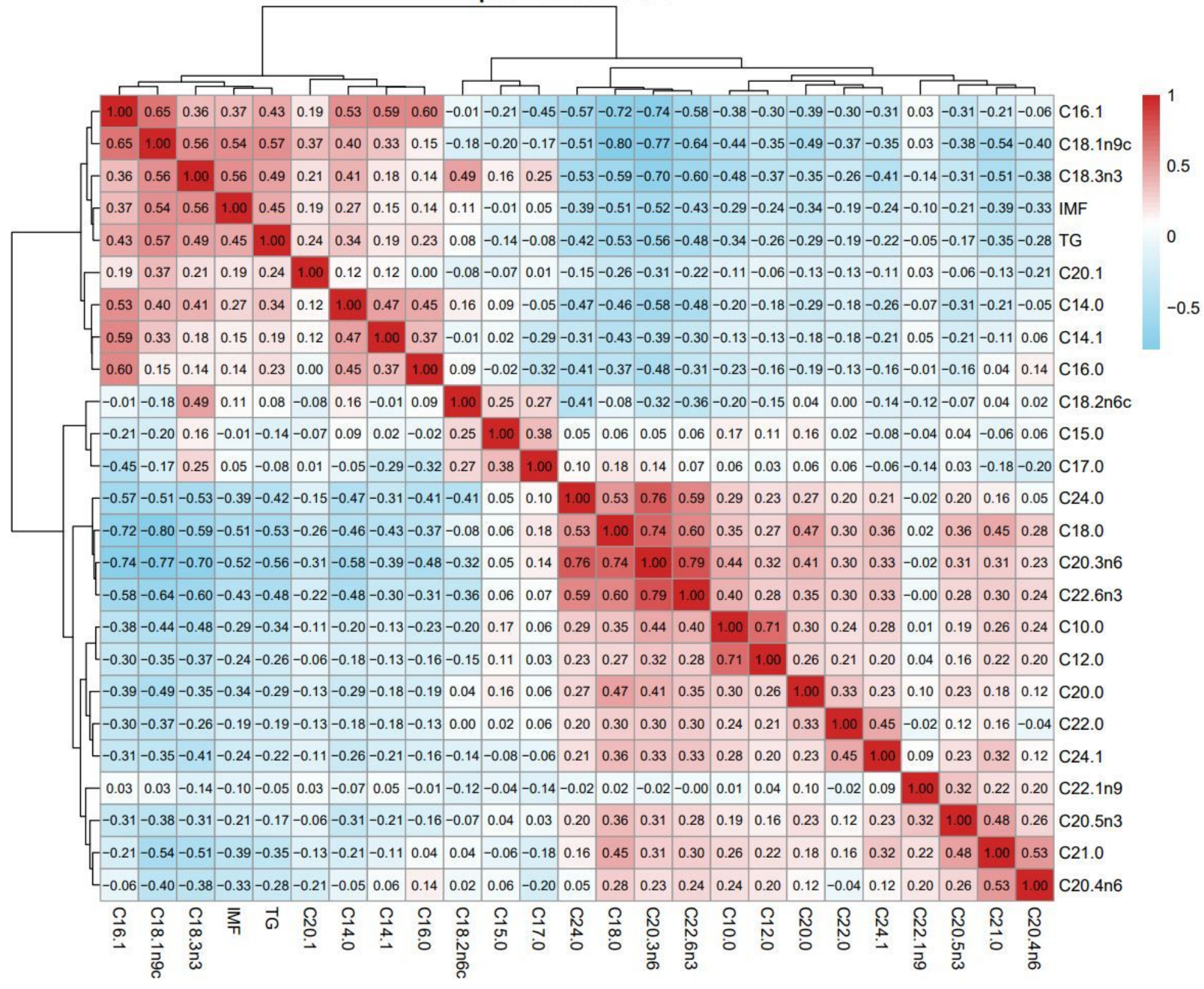

Figure 4

Relationships between IMF, TG, PLIP and fatty acids (FAs). The Spearman correlation analysis was conducted in the R statistics software (version 3.6.1). $n=520$. 


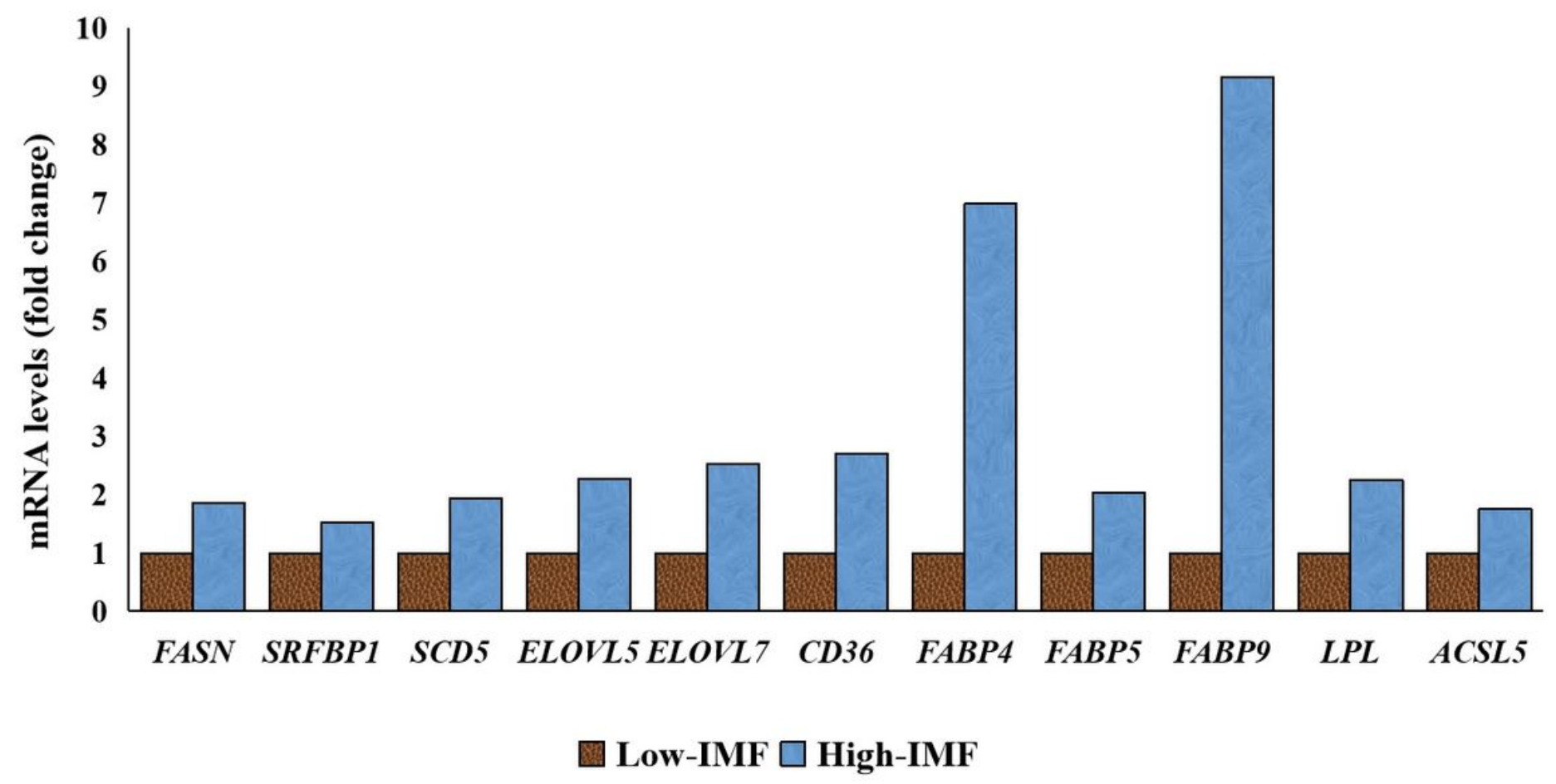

Figure 5

Expression levels of genes related to fatty acid (FA) metabolism in pectoralis of chickens with high- or low-IMF content. Each 8 individuals in the groups with high- or low-IMF content from 520 JXY chickens were used for RNA-sequencing. Genes involved in various processes of FA metabolism, including de novo synthesis (FASN, SRFBP1), release (LPL), desaturation (SCD5), elongation (ELOVL5, ELOVL7), transport (FABP4, FABP5, FABP9, CD36) and activation (ACSL5). Data are expressed as the mean \pm standard error of mean (SEM); $\mathrm{n}=8$. 


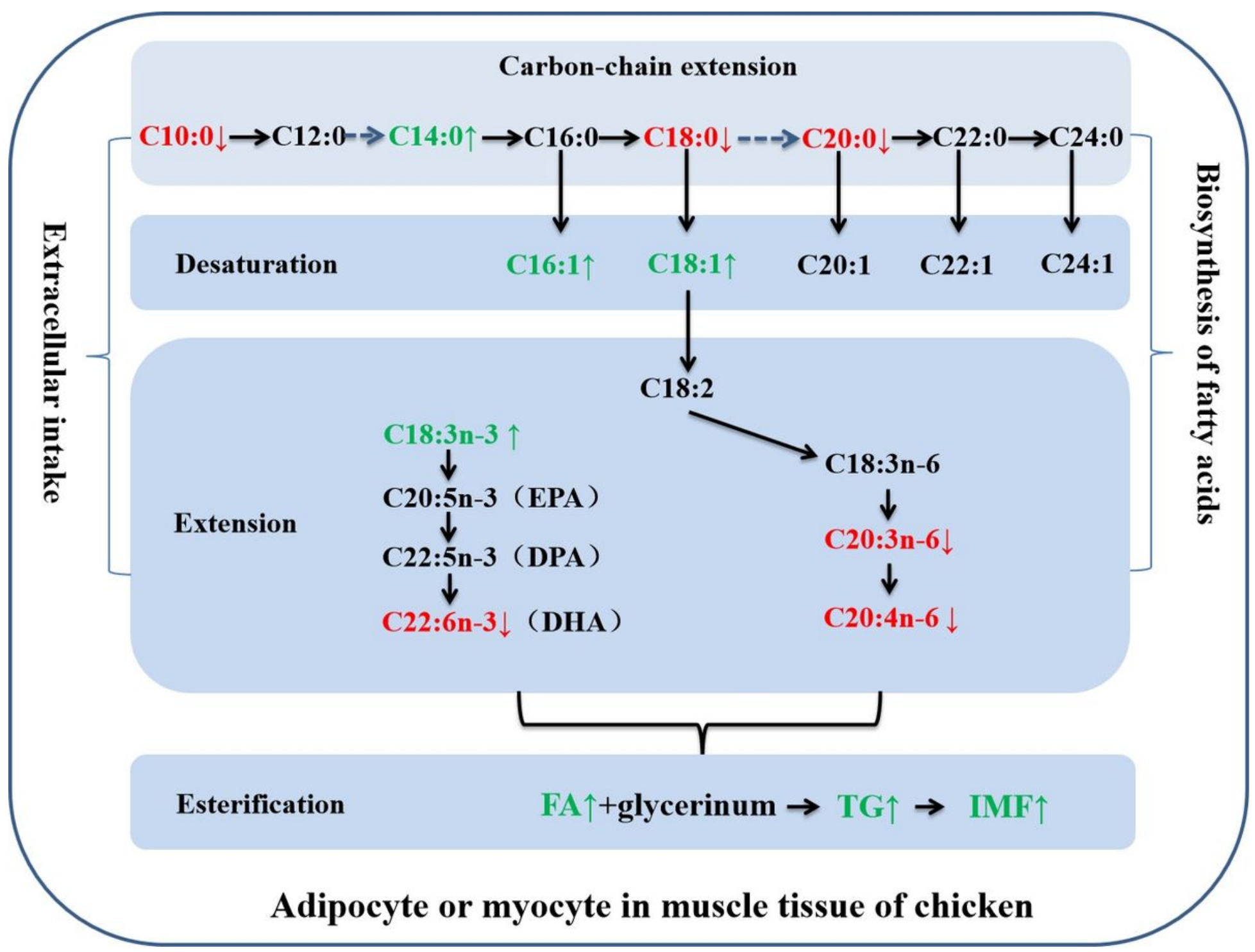

Figure 6

Proposed biochemical mechanism of IMF deposition in chicken meat. This pathway is involved in the biosynthesis of fatty acids (FAs) and triglyceride (TG) in turn. Prevalent accumulation of LCFAs (C16:0, C16:1, C18:1n9c, and C18:2n6c), and inhibitions of MCFAs and VLCFAs would synergistically result in the increase of TGs with the FA biosynthesis and cellular uptake ways, and then accelerate the deposition of IMF in chicken meat.

\section{Supplementary Files}

This is a list of supplementary files associated with this preprint. Click to download.

- SupplementalTable1.xlsx

- SupplementalTable2.xIsx 